\title{
Investigation to Line-Based Techniques for Multi-target Selection
}

\author{
Jibin Yin and Xiangshi Ren \\ Kochi University of Technology, Kochi 782-8502, Japan \\ 088402 e@gs.kochi-tech.ac.jp, ren.xiangshi@kochi-tech.ac.jp
}

\begin{abstract}
This paper presents three selection techniques (called Rubber-lingsweep, Line-string and Coupling-with-pressure) to enhance multi-target acquisition in GUIs and to overcome the drawback of the standard rubber-band box technique, i.e., the limitation of not being able to select an irregular layout of targets. Rubber-line-sweep utilizes a rubber-band line to select targets by "sweeping" them. Line-string employs a line stroke to "string" targets together and select them. Coupling-with-pressure couples these two techniques with pressure as a switch mode. Experiments were conducted to compare these techniques with the standard Rubber-band box, which used a two-dimensional grid which could include varied target sizes, distances and target layouts, and which is applied by using pens as input devices. Experimental results indicate that Rubber-line-sweep, Line-string and Coupling-with-pressure show significant advantages for targets with irregular layouts. Taking performance and subjective ratings together, Coupling-with-pressure outperforms the other three techniques.
\end{abstract}

Keywords: multi-target selection, pen-based interface, pressure.

\section{Introduction}

In user graphic interface (GUIs), fundamental computing operations commonly include single-selection tasks and multiple-selection tasks. Single target selection is usually accomplished by tapping; multiple target selection is usually accomplished using the rubber-band box. The rubber-band box works like this: the rectangular selection region is specified by extending the diagonal of the rubber band box by dragging; the targets interacted by the rectangular selection region are highlighted for selection. An obvious drawback of the rubber-band box is that it is difficult to select the multiple targets that are not included in the rectangular area (see Figure 1c). Conversely, it is impossible to exclude unwanted targets form the rectangular area without further clicks, taps or other maneuvers. So, when selecting multiple targets that are arranged irregularly, the user has to implement a variety of selection tasks such as using tapping the "Ctrl" key and the rubber-band box together. In some sense the rubber-band box limits the user's performance in multiple target selections. Thus we present three novel line-based techniques (Rubber-Line-Sweep, Line-String and Coupling-With-Pressure) to enhance multi-target acquisition. We conducted experiments to compare Rubber-Line-Sweep, Line-String, Coupling-With-Pressure 
and Rubber-band box by pen. In the experiments the selection task was the conjunctive selection of multiple targets (rectangles) in two-dimensional grids varied in terms of the sizes of the targets and layout complexity of targets.

\section{Multi-target Selection Techniques Design}

To overcome the shortcomings of the Rubber band box method we employ rubberband lines or line strokes to select multi-targets instead of the rubber-band rectangle (see Figure 1). (1) Rubber-Line-Sweep: it utilizes rubber-band lines to select targets. Rubber-band lines are very common computer graphic elements, the length and direction of which can be easily adjusted. When the user wants to perform multitarget selection using the Rubber-Line-Sweep he/she first lands the pen-tip on the screen, then moves it to extend a rubber-band line. Targets swept by the rubber-band line are selected. Rubber-Line-Sweep uses "sweep" as the interaction manner of selection. In essence Rubber-Line-Sweep utilizes a rubber-band line to specify a selection area by "sweeping" so it can define irregularly shaped selection areas. To abort selection of a highlighted target the target is swept again by a rubber-band line (see Figure 1a). (2) Line-String: Line-String exploits a line stroke to select targets by stringing them together (see Figure 1b). To abort a highlighted target a line stroke is drawn through the target and the highlighted state is aborted. (3) Coupling-WithPressure: Rubber-line-sweep and Line-string have their own special operational characteristics and advantages. It is even possible to combine the two techniques in special situations, e.g. when they are applied to a pen-based device they can be combined by using pressure as the switch mode between the two techniques, i.e. at light pressure one mode is active while at heavy pressure the other is active. This technique is called Coupling-with-pressure. The threshold value is crucial for achieving a free and stable switch between techniques. If the threshold is very low it is quite likely that the user will unintentionally pass the threshold and change from the currently desired method. Therefore, it should be set to a high value which would require a deliberate change in pressure to switch the operational state. To determine a

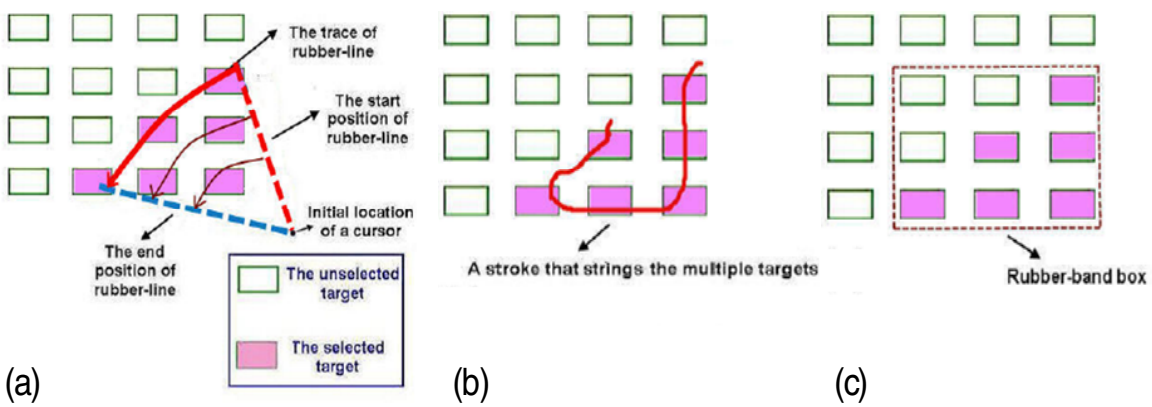

Fig. 1. Selection processes of Rubber-Line-Sweep, Line-String and Rubber-band box: (a) Rubber-Line-Sweep: dragging cursor extends a rubber-band line which is used to sweep targets to select them; (b) Line-String: drawing a stroke to string targets to select them.(c) an irregular layout of targets can not be completely included by a rectangle. 
proper switch threshold a pilot experiment with 10 subjects was performed. The task was to draw freehand strokes (arbitrary curves and straight lines), basic geometrical graphs (such as rectangles and circles) and a mixed set of Roman, Japanese, and Chinese characters (kanji) and signatures on a blank space in a natural manner. Pentip pressure was recorded in a $17 \mathrm{~ms}$ sampling periods. Ninety-five percent of the force samples fell within the 210 to 810 units range. The results showed that the pressure level of more than 810 units was seldom used in a natural manner. Therefore, in Coupling-with-pressure the threshold value is set at 970 units. Visual feedback which denotes the current pressure value is added to Coupling with pressure. Ramos et al.' study [6] shows that a good visual feedback is needed for pressure-based UI design. Inspired by the pressure cursor design by Ramos and Balakrishnan [5], in Coupling-with-pressure, pressure was displayed in a wedge-shaped graphical widget. The transparent green area indicates the current pressure value. The top border represents the switch threshold. When pressure is beyond the top border the technique is changed from Rubber-line-sweep (default status) to Line-string.

\section{Experiment}

We explored the performance efficiency of the four multi-target selection techniques in different circumstances. The complexity of the layout of the targets and the number of targets were varied.

Twelve volunteers (10 male and 2 female) participated in Experiment. The average age was 24.3 years (ranging from 21 to 31 ). All were right-handed. The hardware used in Experiment was a Wacom DIT-520 interactive LCD graphics display tablet with a wireless stylus that has a pressure sensitive isometric tip, which reports 512 levels of pressure and has a binary button on its barrel.

\subsection{Procedure}

Conjunctive multi-target selection tasks are prescribed in Experiment. For each trial, thirty-six squares were shown on a $6 \times 6$ grid. Target squares were green in color, and the other (non-target) squares were white with a blue frame. With respect to the number of targets in a task, there are three types of tasks: four target tasks, nine-target tasks and sixteen target tasks. With reference to target layout, we provided three complexity degrees for the tasks: low complexity tasks, medium complexity tasks and high complexity tasks, which were determined by the subjects in pilot study. Thirtysix different target layouts were used in Experiment.

\subsection{Results}

For Low and Median complexity layout of targets repeated measures analysis of variance showed no significant difference in selection time between the four selection methods. For High complexity layout there was a significant difference in selection time between the four selection methods, $\mathrm{F}(3,43)=6.3, \mathrm{p}<.01$. The post hoc Tukey HSD test showed that Rubber-lines weep, line-string and Coupling-with-pressure were all faster than Rubber-band box $(\mathrm{p}<.05)$. There were no other significant differences across the selection methods. Coupling-with-pressure was the fastest 
method followed in older by Line-string, Rubber-line-sweep and Rubber-band box. No significant difference was found between the four selection methods in error rate.

Subjects gave Coupling-with-pressure the highest rating followed in order by Linestring, Rubber-line-sweep and Rubber-band box. Some subjects mentioned that Coupling-with-pressure was more flexible and it enabled the user to choose a proper method (between Rubber-line-sweep and Line-string) depending on the conditions of the selection tasks at any particular time.

\section{Discussion}

In this study we present three techniques to enhance multi-target selection, especially for irregular layouts of targets. Experiment results suggest that Rubber-line-sweep, Line-string, and Coupling-with-pressure are all faster than Rubber-band box for complex layouts of targets. They are all comparable to Rubber-band box for simple and median layouts of targets. Overall, Coupling-with-pressure is the fastest technique and the most preferred by subjects. Pressure, as an additional input parameter, is seldom explored and applied into UI designs. Coupling-with pressure offers a promising instance of pressure-based applications. We believe that the results of our work uncovered several basic principles that are applicable directly toward the design of interaction techniques for multi-target acquisition, particularly in pen-based interfaces.

\section{References}

1. Accot, J., Zhai, S.: More than dotting the i's - foundations for crossing-based interfaces. In: Proc. of CHI'04, pp. 73-80 (2004)

2. Chipman, S.F.: Complexity and structure in visual patterns. Journal of Experimental Psychology: General 106(3), 269-301 (1977)

3. Miller, R., Myers, B.: Multiple Selections in Smart Text Editing. In: Proc. of IUI'02, pp. 103-110 (2002)

4. Mizobuchi, S., Yasumura, M.: Tapping vs. Circling Selections on Pen-based Devices: Evidence for Different Performance-Shaping Factors. In: Proc. of CHI'04, pp. 607-614 (2004)

5. Ramos, G., Balakrishnan, R.: Zliding: Fluid Zooming and Sliding for High Precision Parameter Manipulation. In: Proc. UIST'05, pp. 143-152 (2005)

6. Ramos, G., Boulos, M., Balakrishnan.: Pressure Widgets. In: Proc. CHI'04, pp. 487-494 (2004) 